\title{
Multimodal Molecular Imaging: Current Status and Future Directions
}

\author{
Min Wu $(\mathbb{D}$ and Jian Shu $(10$ \\ Department of Radiology, The Affiliated Hospital of Southwest Medical University, Luzhou, Sichuan, China \\ Correspondence should be addressed to Jian Shu; shujiannc@163.com
}

Received 17 January 2018; Revised 11 April 2018; Accepted 10 May 2018; Published 5 June 2018

Academic Editor: Giancarlo Pascali

Copyright ( $) 2018 \mathrm{Min}$ Wu and Jian Shu. This is an open access article distributed under the Creative Commons Attribution License, which permits unrestricted use, distribution, and reproduction in any medium, provided the original work is properly cited.

\begin{abstract}
Molecular imaging has emerged at the end of the last century as an interdisciplinary method involving in vivo imaging and molecular biology aiming at identifying living biological processes at a cellular and molecular level in a noninvasive manner. It has a profound role in determining disease changes and facilitating drug research and development, thus creating new medical modalities to monitor human health. At present, a variety of different molecular imaging techniques have their advantages, disadvantages, and limitations. In order to overcome these shortcomings, researchers combine two or more detection techniques to create a new imaging mode, such as multimodal molecular imaging, to obtain a better result and more information regarding monitoring, diagnosis, and treatment. In this review, we first describe the classic molecular imaging technology and its key advantages, and then, we offer some of the latest multimodal molecular imaging modes. Finally, we summarize the great challenges, the future development, and the great potential in this field.
\end{abstract}

\section{Introduction}

Molecular imaging has emerged at the end of the last century and consists of a combination of in vivo imaging and molecular biology aiming at identifying or describing living biological process at a cellular and molecular level using noninvasive procedures. It is especially addressed to reveal abnormalities in cells and molecules which cause the disease, rather than the final anatomical and structural abnormality caused by cellular or molecular changes [1]. Various modern imaging technologies have been widely used to monitor structural, functional, and molecular changes in cancer tissues, including optical imaging (either by bioluminescence or fluorescence) [2], computed tomography (CT) [3], magnetic resonance imaging (MRI) [4], positron emission tomography (PET) [5], single-photon emission computed tomography (SPECT) [6], and ultrasound (US) [7]. Molecular imaging can detect lesions and determine the nature of earlier lesions more accurately compared to conventional imaging, so that clinicians can effectively intervene in the occurrence and formation stage of the disease [8]. In recent years, with the appearance and development of molecular imaging, early tumor diagnosis became possible.
Consequently, the latest advances in multimodal molecular imaging are reviewed in this paper.

In clinical research, CT, MRI, PET, SPECT, US, and optical imaging are usually among the choices of the imaging modalities. Each imaging modality has its own unique strength and intrinsic limitations, such as spatial/depth resolution and sensitivity, making the achievement of precise and reliable information at the disease site difficult. In order to compensate these weak aspects, multimodal molecular imaging has been considered in recent years [9]. Multimodal molecular imaging can play important roles in the clinical care of various diseases by improving clinicians' ability to perform screening, surveillance, staging, prognosis, planning and therapy guidance, monitoring therapy efficacy, and assessing recurrence. With its rapid technological advances, presymptomatic detection, targeted therapy, and personalized medicine may be possible in the near future through the use of multimodal molecular imaging.

\section{Molecular Imaging Models}

At present, molecular imaging models mainly include CT, MRI, radionuclide, ultrasound, and optical imaging. 
2.1. CT Molecular Imaging. CT is a technique producing images reflecting human anatomy, thanks to differential levels of X-ray attenuation by tissues within the body. CT is widely available, and it has some advantages such as high spatial resolution, strong penetration depth, fast acquisition time, low cost, clinical utility, and relative simplicity. However, CT also has some disadvantages: one is the high radiation dose, which often limits the scan time, and another is the low-quality soft tissue contrast, compared with MRI [10]. In addition, there is the low sensitivity of current CT contrast agents. It is indeed valuable in identifying and assessing several diseases including tumors, brain injury, and pulmonary embolism.

The application of CT molecular imaging requires high quality imaging agents, after injection, in order to achieve the target site for a change in X-ray attenuation. Currently, most CT molecular contrast agents are designed to combine a maximum number of X-ray-absorbing atoms with a nanoparticle, which includes emulsion, liposomes, lipoproteins, and polymeric nanoparticles [11-17].

Until now, multiple examples of the abovementioned CT molecular imaging have started to appear, and there has been a major development in CT molecular imaging. In 2006, Rabin et al. [18] first reported the synthesis of a polymer-coated bismuth sulfate $\left(\mathrm{Bi}_{2} \mathrm{~S}_{3}\right)$ nanoparticle as an injectable CT imaging agent, which is used for enhanced in vivo imaging of the vasculature, the liver, and lymph nodes in normal mice. Next, Hyafil et al. [19] reported cellular imaging using CT in atherosclerotic plaques in rabbits using an iodinated nanoparticle dispersed with the surfactant. $\mathrm{Li}$ et al. [20] reported the use of a 2-deoxy-d-glucose- (2-DG-) labeled gold nanoparticle (AuNP-2-DG) for targeted molecular CT imaging in malignant neoplasms to obtain highresolution metabolic and anatomic information. Kim et al. [21] reported the synthesis of CT-compatible gold nanoparticles optimized with prostate-specific membrane antigen RNA aptamers and the use of CT molecular imaging and therapy of prostate cancer. Furthermore, Kayyali et al. [22] reported the use of targeted gold nanoparticles and liposomal iodine, respectively, as contrast agents for inner ear imaging, and the results showed that significant enhancement of micro-CT images was observed using liposome iodine. Choi et al. [23] reported the preparation of X-ray CT/US dual-modal imaging probe (GC-DTA-PFP NPs), and the study indicated that X-ray CT/US dual-modal imaging could provide more comprehensive and accurate diagnostic information about the diagnosis of tumor. Yue et al. [24] developed a $\mathrm{pH}$-responsive multifunctional nanotheranostic agent (FePt/GOCNs) for potential in vivo and in vitro dualmodal MRI/CT imaging and in situ cancer inhibition.

The applied research of CT molecular imaging is becoming more and more widespread, and the reason is that they have the potential for dose reduction as low as possible while enhancing image contrast [25], display an X-ray attenuation property better than commercial iodinated smallmolecular-contrast agents [26], show much stronger CT imaging effect compared with the traditional small molecule contrast agents [27], and have CT cell-tracking applications for noninvasive monitoring $[3,28,29]$. In short, although
CT molecular imaging abilities have not yet been completely explored, it remains an extremely useful morphological tool. When associated with other imaging modalities, CT also can give an anatomical reference frame for the biochemical and physiological findings that are afforded by these other imaging instruments [30].

2.2. MRI Molecular Imaging. Today, MRI is seen as the most useful imaging modality in radiology, especially in the detection and characterization of soft tissue pathology [31, 32]. MRI can provide three-dimensional clear images, it has high spatial resolution and high contrast, and the acquisition time and the quality of images are constantly improving, thanks to technological innovations [32]. These aspects are described below by some specific MRI methods.

From a great many studies, including work in packed cells, it is easy to see that cell density varying inversely is reflected in conventional measurements of diffusion using MRI report values of ADC. Consequently, DWI has been used to evaluate tumor cellularity $[33,34]$. A quantitative map that acquired tumor cellularity in vivo may be a useful tool for both treatment planning and monitoring. Early studies revealed abnormal water diffusion in various tumors, and more detailed quantitative relationships have always been explored recently between microstructure and ADC. Zhao et al. [35] discovered the ADC changes in response to tumor treatment by measuring water ADC in excised RIF-1 tumors after treatment with an anticancer drug. These data indicated that ADC increase began while the tumors were still growing and implied that it could be an early indicator of valuable cytotoxic treatment response. Hereafter, Henning et al. [36] reported the value of $\mathrm{ADC}$ for quantitative assessments of individual tissue regions, tumor growth kinetics, and cell kill in RIF-1 tumor animal models. Moreover, in the related studies, higher pretreatment ADC values incline to correlate with poorer response to therapy and prognosis [37-39]. For example, one of these studies shows that, on performing DWI in hepatic metastases, it appears that $\mathrm{ADC}$ values are effective on predicting and monitoring the early chemotherapeutic response of these metastases which originated from gastrointestinal tumors [39]. In these patients, the pretherapy mean ADC value in nonresponding lesions is significantly higher than that in responding lesions. Similarly, Koh et al. [38] found that higher pretreatment ADC values were predictive of poor response to chemotherapy in a small group of patients with colorectal hepatic metastases.

The examples above demonstrate the usefulness of indirect approaches to measure molecular changes in tissues, but many other researchers preferred the approach of a direct molecular imaging using MRI. At present, there are two major types of MRI molecular imaging probes: one is the direct detection of a nuclear species, that is, a component of an imaging probe (e.g., nuclei detection with the use of ${ }^{31} \mathrm{P}$, ${ }^{23} \mathrm{Na},{ }^{19} \mathrm{~F},{ }^{1} \mathrm{H}$, or ${ }^{13} \mathrm{C}$ within molecules introduced into the body), which is realized by magnetic resonance imaging (MRS), and the other approach consists of an indirect detection via the effects of an agent on the large signal originated from the hydrogen nuclei (protons) in tissue water, 
either by changing the water relaxation rate or by introducing new pathways for magnetization transfer [40].

For the first of direct approaches, MRS has been widely used to detect metabolic changes in cancerous as well as in normal tissues. MRS can not only provide information on biochemical changes in response to tumor growth but also delineate different metabolic tumor phenotypes. For instance, ${ }^{1} \mathrm{H}$ MRS is widely used to monitor metabolic changes in cancer tissue [41-47], and the other active nuclei such as ${ }^{31} \mathrm{P}$ (phosphorus) $[48,49],{ }^{23} \mathrm{Na}$ (sodium) $[50],{ }^{13} \mathrm{C}$ (carbon) [51-53], and ${ }^{19} \mathrm{~F}$ (fluorine) $[54,55]$ are also being used to monitor bioenergetics and metabolic in cancer.

For the second of direct approaches, MRI implies the use of paramagnetic or superparamagnetic agents that alter the tissue proton relaxation time $\mathrm{T} 1, \mathrm{~T} 2$, or $\mathrm{T} 2{ }^{*}$ or manipulate the magnitude of the water signal via specially designed radiofrequency irradiation that labels one species of protons that in turn transfers the label to the water via magnetization exchange [56]. For example, Ta et al. [57] reported the development of functional multimodal iron oxide nanoparticles for targeted MRI in atherosclerosis, which uses a combination of chemical and biological conjugation techniques. The ultrasmall magnetic dual-contrast iron oxide nanoparticles are used to be efficient positive and negative dual-contrast agents for magnetic resonance imaging and are also labeled with fluorescent molecules to allow for optical imaging.

So far, the design of MR contrast agents may be divided into four different types for different applications. The first is represented by nonspecific contrast agents. For example, the commonly used lanthanide chelates or intravascular blood pool agents [58] are both missing the ability to reach specific targets. Low-molecular-weight Gd(III) complexes have become an essential tool in the detection and characterization of many diseases [59]. The second is represented by targeted contrast agents. They are usually paramagnetic species glued to or part of specifically molecules, such as antibodies that are directed toward and taken up by specific molecular targets [60-62]. The third is represented by so-called "smart" contrast agents. Although they do not rely on selective targeting to achieve spatial specificity, they change their efficacy only in response to specific local molecular characteristics, such as the presence of specific proteinases, or changes in environmental $\mathrm{pH}$ [63-67]. The fourth is represented by labeled cells. They can be bound to or introduced into specific cell types such as T cells or stem cells, which then rely on the trafficking and recognition of the cells for their localization [68-71].

The above four types of MR contrast agents can be all used in different aspects of MRI molecular imaging. Furthermore, Ko et al. and Yang et al. [72, 73] reported the construction of PET/MRI dual-mode probe, which can perform PET and MRI imaging at the same time of the tumor. Kircher et al. [74] further developed a new triplemodality MRI-photoacoustic-Raman nanoparticle (MPR nanoparticle) to perform multimodal molecular imaging, and the three-mode molecular probe can obtain more accurate brain tumor resection by exploiting the complementary strengths of each modality.
Overall, magnetic resonance imaging is one of the most important molecular imaging research methods, which can be used for noninvasive monitoring and early diagnosis of diseases at the cellular and molecular level. In recent years, the study of magnetic resonance molecular imaging is increasing and is mainly used for cell tracking, angiogenesis, apoptosis, and in vivo tissue gene imaging. Although the techniques still have some problems that need an urgent solution, its unique advantages make its application prospect worthy of expectation in clinical medicine and basic research.

\subsection{Radionuclide Molecular Imaging. Radionuclide imaging} is one of the four major medical imaging techniques, and it is a radioactive marker in drug, when the body organs and tissue absorb and form radiation source in the body, and then, the nuclide detection device can be used to detect isotope in the process of decay on the rays, which constitute the image of radioactive isotopes in in vivo distribution density $[75,76]$. In recent years, with the rapid development of molecular biology and nuclear medicine technology, the field of nuclear medicine has formed a new branch of nuclear medicine-molecular nuclear medicine [77]. SPECT and PET are advanced radionuclide molecular imaging techniques that are able to evaluate biochemical changes and levels of molecular targets within a living subject. Both techniques enable whole body imaging of molecular targets/processes with high sensitivity. SPECT is mainly used for whole body bone imaging [78-80], myocardial blood flow imaging [81-84], cerebral blood flow imaging [85-89], and thyroid imaging [90-95]. PET is mainly used to detect dynamic changes in the metabolic function of substances (or drugs) in the human body, and it widely used for the nervous system, the cardiovascular system, and the oncology [96-101].

The imaging agents used for PET are the basic elements for the human body, used to easily mark compounds and metabolites, and do not change their biological activity, so as to reflect the molecular level of physiological and biochemical processes of the human body, to achieve the purpose of early diagnosis and guidance of treatment. In clinical practice, PET that enables to locate, stage, and monitor cancer is mainly used to image tumors through the use of the 18F-labeled imaging agent [18F]-2-fluoro2-deoxy-glucose ([18F]-FDG) [102-104]. For instance, Brenner et al. [105] reported that the predictive values of $[18 \mathrm{~F}]-\mathrm{FDG}$ in primary staging in patients with newly diagnosed nonseminomatous germ cell tumor (NSGCT) clinical stage I/II. Kindred et al. [106] investigated glucose uptake asymmetries in leg muscles of patients with mild multiple sclerosis (MS) during walking with a glucose tracer (18F-FDG),and the result showed that [18F]-FDG uptake was significantly lower in the weaker knee flexors of patients with MS. Winther-Larsen et al. [107] evaluated whether changes in [18F]-FDG uptake evaluated early during erlotinib treatment predict survival in nonsmall-cell lung cancer (NSCLC) patients.

In addition to its clinical practicality, PET has extensive applications in the basic and preclinical researches. PET can be used to study basic physiological and molecular 
mechanisms of human diseases by using the appropriate radiolabeled-imaging agents [108]. Bretin et al. [109] reported that the biodistribution of the PET tracer over time can be determined in vivo. Moreover, PET can be employed for the evaluation of novel radiolabeled-PET imaging agents, biodistribution of novel pharmaceuticals in suitable animal models, and effectiveness of new therapies $[30,110]$. For instance, Nanni et al. [111] studied small animal PET for the early detection of malignant masses in a xenograft murine model of human rhabdomyosarcoma and analyzed the metabolic behavior of this xenograft tumor over time.

SPECT imaging agents use energy between 85 and $500 \mathrm{Kev}$; radiographic tomography is a technique for projection reconstruction of faulty images, which are similar to X-ray and CT imaging. SPECT uses nuclides such as ${ }^{99 \mathrm{~m}} \mathrm{Tc}$ $[112-114]$, and ${ }^{123} \mathrm{I}[115,116]$ through the emission of single gamma rays decay to obtain different energies. SPECT is one of the most commonly used nuclear medicine modalities in clinical practice [117]. Some examples of clinical use are the potential usefulness of ${ }^{99 \mathrm{~m}} \mathrm{Tc}$-TRODAT-1 imaging in the evaluation of patients with early-stage Parkinson's disease [118], the therapeutic effects of ${ }^{111}$ In-DTPA-octreotide in tumors of various sizes [119], and the location and excision of the tumor [120]. Besides, small-animal SPECT is designed for imaging small animals specifically [121, 122], and it has been used for many preclinical studies. To name a few, Wang et al. [123] studied that the dynamics and feasibility of imaging nonsmall-cell lung cancer (NSCLC) apoptosis induced by paclitaxel treatment using ${ }^{99 \mathrm{~m}} \mathrm{Tc}$-labeled C2A domain of synaptotagmin I in a mouse model. Moscaroli et al. [124] presented an ${ }^{111}$ In-radiolabeled FGF-2 derivative for noninvasive imaging in small animals deploying singlephoton emission tomography (SPECT). Tang et al. [125] developed a radiolabeled tyrosine kinase inhibitor (TKI) for HER2-targeted breast cancer imaging.

Since biochemical changes always occur before anatomical changes in disease, both PET and SPECT have clear diagnostic strength over anatomical techniques such as classical CT and MRI. However, PET and SPECT have a key weakness, that is, the lack of an anatomical reference frame. This weakness may be eliminated through the combination of these instruments with either CT or MRI, producing a single scanner capable of accurately identifying molecular events with precise correlation to anatomical findings [126]. This method is known as "multimodality imaging," in which two or more modalities are used in combination to develop their individual strengths and compensate for the weaknesses of each imaging system. For example, Glaus et al. [127] developed a novel nanoparticle-based dual-modality positron emission tomography/magnetic resonance imaging (PET/MRI) contrast agent; the probe produced strong MR and PET signals and were stabled in mouse serum for $24 \mathrm{~h}$ at $37^{\circ} \mathrm{C}$. Chen et al. [128] synthetized folate-NOTA- $\mathrm{Al}^{18} \mathrm{~F}$ radiotracer and examined its properties both in vitro and in vivo, for PET imaging of folate receptor-positive tumors. Xing et al. [129] built a dual-mode probe which uses RGD as a target probe and quantum point as the carrier. Then, this dual-mode probe is covered with PEG to improve water solubility and is linked to DOTA which chelates ${ }^{64} \mathrm{Cu}$. Thus, the nuclear medical imaging is associated with near-infrared imaging. Finally, the authors show that the PET signal is highly coincided with quantum dot near-infrared image.

So far, nuclear medicine molecular imaging technology is one of the widely used technologies in clinical molecular imaging technology and plays an important role in the study of personalized medical care due to its unique technology [130-133]. PET and SPECT are not only powerful tools for basic medicine and pharmacy but also the best tools for detecting and guiding the treatment of various diseases and tumors $[110,134,135]$. They contribute to developing treatment programs on the tumor and other diseases, which are made by clinicians more scientific, more comprehensive, and more reasonable. Their application will have a profound impact on clinical practice.

2.4. Ultrasound Molecular Imaging. Ultrasound imaging, like MRI and CT, has been used as a morphological imaging modality. Medical ultrasound imaging is a unique imaging modality that exploits the properties and behavior of high-frequency sound waves as they travel through biological tissue, and it can be used both for diagnostic imaging and as a therapeutic tool. Compared with traditional imaging techniques such as radionuclide imaging and optical imaging, ultrasound imaging has some advantages such as economy, convenience, and real-time imaging [136, 137]. Furthermore, ultrasound molecular imaging of contrast agents which combine with the target organ can be used as a carrier for therapeutic drugs or genes, so as to achieve a multiplier effect [138-140].

Traditional ultrasound contrast agents, that are a few micrometers in diameter and in the terms of gas-filled microbubbles, are often coated with lipids or biopolymers, and they are available for enhancing the reflection signal-to-noise ratio for blood [141]. These contrast agents have provided useful imaging data, but they do not enable imaging of specific molecular events. However, by attaching certain antibodies [142-144], peptides [145, 146], or other targeting moieties [147] to the surface of microbubbles, those particles can target specific biochemical processes to achieve ultrasound molecular imaging. Ultrasound molecular imaging that uses in vivo simulation of immunohistochemistry or in situ hybridization techniques targets biomolecules to highlight the pathological changes of diseased tissue. Thus, it can visualize the real pathogenesis and significantly improve the sensitivity and accuracy of imaging diagnosis. These aspects are actually the current clinical research central issues.

At present, targeted microbubbles are being used in preclinical investigations of both inflammation and angiogenesis. For example, microbubble shells have been attached to endothelial cell adhesion molecules for visualization of P-selectin, supplying foresight on molecular aspects of inflammation [148]. Deshpande et al. [149] showed that P-selectin-targeted microbubbles (MBs) can be used to monitor the expression of this molecule as a marker of inflammation in a murine model of inflammatory bowel disease (IBD). In order to target vascular endothelial growth 
factor receptor-2 (VEGFR2), Willmann et al. [150] constructed anti-VEGFR2 antibodies attached to microbubbles. After using either targeted microbubbles or control microbubbles in tumor-bearing nude mice, ultrasound imaging studies can be performed. Compared with studies using only control microbubbles, imaging results demonstrated a significantly higher average intensity in images from studies using targeted microbubbles. Liu et al. [145] developed endothelial-targeted microbubbles (MBs) and employed targeted microbubble-enhanced ultrasound (US) imaging to assess the endothelial expression levels in neovasculature for noninvasive assessment of colorectal tumor angiogenesis.

With the emergence of ultrasound molecular imaging, the early diagnosis and specific treatment of malignant tumors gained some research achievements. Kim et al. [151] used ink-containing PLGA polymer microbubbles to achieve both enhanced optical and ultrasound imaging of breast cancer with a depth of less than $18 \mathrm{~mm}$. Cochran et al. [152] study showed that injecting paclitaxel microbubbles can effectively avoid acute poisoning reaction caused by direct injection of paclitaxel, thus significantly reducing paclitaxel side effects and effectively inhibiting tumor growth. Wang et al. [153] reported that integrin-targeted nanoparticle gene vector can specifically act on vascular endothelial cells of mice tumors, thus inducing tumor regression. Liu et al. [154] prepared perfluoropentane nanodroplets modified by folate and encapsulated by lipid membrane (FA-NDs) and investigated the nanodroplets stability in different temperature and its target performance of SKOV3 tumor cells in vitro and in vivo. Hu et al. [155] verified that tumor over expression of SHP2 and other protein tyrosine phosphatases regulated several cellular processes and contributed to tumorigenesis, which could be introduced to ultrasound molecular imaging for differentiating normal from malignant thyroid diagnostic nodes.

In the near future, ultrasound molecular imaging is expected to pass from a preclinical modality to a fully clinically useful technique through the use of different clinically translatable instrumentation, such as endoscopes and novel US-compatible imaging agents that are able to exudate $[156,157]$. Among the agent-based molecular imaging techniques, targeted ultrasound imaging has promising huge developments.

2.5. Optical Molecular Imaging. Optical imaging is a method of obtaining biological information by using optical detection means combined with optical detection molecules to imaging cells or tissues or even organisms. If the biological optical imaging is limited to the visible and near-infrared range, different biological optical imaging methods can be divided into fluorescence imaging, bioluminescence imaging, photoacoustic imaging, and optical tomography. Nowadays, molecular imaging becomes more popular and is combined with classical optical imaging techniques.

Fluorescence imaging technology is marked with a fluorescent report group including inorganic materials, such as upconversion, quantum dots, and other organic materials, such as green fluorescent protein, red fluorescent protein, or fluorescent dye. It uses excitation light to make the report group reach a higher level of molecular level and then emit a longer wavelength visible light to form biological light source in vivo and detect it. At present, common fluorescent groups include various small molecule fluorescent dyes, green fluorescent protein, and red fluorescent protein. In recent years, fluorescence technology has been extensively used in the study of molecular biology and the metabolism of small molecules in small molecules. There is a rapidly expanding list of fluorescent agents which includes near-IR Cy 5.5, turnip green, quantum dots (QDots), the Alexa dye series, and all kinds of fluorescent proteins. Besides, lanthanide-based imaging agents were added to this list [158]. There are numerous strengths of these agents which are better than the aforementioned dyes and proteins. They have narrow, nonoverlapping emission bands, long luminescence lifetimes, and allow multiplexed quantitative measurements of the intracellular analysis concentrations $[158,159]$. Fluorescence molecular tomography (FMT) has been applied to visualize and quantitate a variety of cellular and molecular events. In opposition to planar fluorescence imaging, FMT can produce quantitative information and allows imaging at greater depths, up to several centimeters [10]. In 2009, Hyde et al. [160] first used FMT/CT dual-mode imaging to observe the distribution of the focal region of the brain in the mouse model of Alzheimer's mice. Lin et al. [161] developed a FMT/MRI fusion imaging system for small animal imaging, which verified the accuracy of FMT imaging provided by the anatomical structure provided by MRI.

Bioluminescence imaging technology uses luciferase gene to label cells or DNA and exploits sensitive optical detection instrument to directly monitor cell activity and gene behavior in living subjects. This technique has these following advantages: (1) noninvasive, (2) continuous repeated detection, (3) fast real-time scanning imaging, and (4) high sensitivity. Bioluminescence imaging has been used to study numerous enigmatic protein-protein interactions. One such study uses a firefly luciferase-based protein fragment complementation assay to visualize luciferaseexpressing bone marrow cells in brain inflammation in living mice [162]. In 2006, Wang et al. [163] built a separate BLT/CT dual-mode imaging system, BLT data acquisition system to the mice in the imaging process, and then saved the mouse position and posture, and the acquisition of CT data, finally anatomical information obtained from CT into BLT reconstruction, so as to improve the accuracy of BLT imaging.

Photoacoustic imaging (PA) by using optical absorption and transformation between the tissues of the light and sound energy is a nondestructive imaging method developed in recent years. It combines the high penetration characteristics of pure optical imaging and high contrast characteristics by light into the ultrasound, and it can provide tissue imaging with high resolution and high contrast. Based on the technology of photoacoustic effect of time-domain photoacoustic spectrum, it partially overcomes the effect of strong scattering in the optical transmission in the organization when optical and acoustic are organically combined. 
Therefore, photoacoustic technology has well biological penetration, the characteristics of high resolution, and no side effects. Its main application direction can effectively carry out biological tissue structure and function imaging, providing an important means for studying the morphological structure, physiological characteristics, pathological characteristics, and metabolic function of biological tissue. For example, Ding et al. [164] developed a novel contrast agent where the surface of superparamagnetic iron oxide (SPIO) nanoparticles is functionalized with a bladder cancer-specific fluorescein isothiocyanate- (FITC-) labeled cell penetrating peptide- (CPP-) polyarginine peptides (R11) for active targeting and imaging. The results indicate great potential of SPIO-R11 as a contrast agent to target bladder cancer for diagnostic and therapeutic applications.

At present, there are still some defects in the living organism imaging system. Many in vivo optical imaging are also just stay in the phantom and small animal experimental stage, has not yet entered into clinical application, and need a further improvement in many aspects. It is an important task for the future to find new high quantum efficiency fluorophores, improve the reconstruction algorithm and image resolution, and expand the new optical imaging technology. In vivo bioluminescent imaging technology has become an indispensable tool in the research of nuclear small-animal models. It studies the pathological process, drug development, and drug efficacy from a unique perspective. In fact, the biological optical imaging technology has had a significant impact on the basic and applied medical research.

\section{Application of Multimodal Molecular Imaging}

Multimodal molecular imaging combines two kinds or more detection technologies to form a new way of imaging, which is convenient for obtaining some further information in diagnosis, treatment, and monitoring. At present, multimodal molecular imaging has been widely used to optimize medical research and clinical practice. In practice, multimodal molecular imaging has been helpful for cardiovascular diseases $[165,166]$, neuropsychiatric diseases [167-170], and other clinical diseases [168, 171-175]. In addition, it can significantly enhance the positioning of the tumor border and effectively guide the surgical resection of the tumor $[74,176,177]$.

A few specific examples are as follows: (1) cardiovascular diseases: Yoo [178] presented multimodal intravascular optical imaging combining optical coherence tomography and fluorescence lifetime imaging. It can provide new opportunities to investigate vascular pathobiology and diagnose cardiovascular disease, by simultaneously visualizing plaque morphology and biochemical composition; (2) neuropsychiatric diseases: Voss et al. [179] used multimodal functional-imaging technology to study a patient with marked neurological recovery after cranioplasty, and the results suggested resting-state networks and auditory responses obtained with functional MRI and cerebral metabolism obtained with PET before and after cranioplasty revealed significant functional changes that were correlated with the subject's neurological recovery. Wang [180] studied the multimodal imaging investigation of brain mechanisms in neuropsychiatric disorders, emphasizing on the research questions of whether and how neurochemistry is associated with brain anatomical structures and brain functions; and (3) other clinical diseases: Tang et al. [175] developed a novel multimodal video endoscope and evaluated its usefulness for the early detection of gastric neoplastic lesions; the imaging platform is a modified upper GI endoscope capable of whitelight imaging (WLI), wide field vital-dye fluorescence imaging (VFI), and high-resolution microendoscopy (HRME) in a single endoscopic insertion.

In summary, multimodal molecular imaging has a bright future. The development of this field will bring a major breakthrough in medical imaging and molecular biology. Although molecular imaging remains at its initial stage, a broader space for further developments is still possible.

\section{Conflicts of Interest}

The authors declare that they have no conflicts of interest.

\section{References}

[1] R. Weissleder and U. Mahmood, "Molecular imaging," Radiology, vol. 219, no. 2, pp. 316-333, 2001.

[2] C. G. Hadjipanayis, H. Jiang, D. W. Roberts, and L. Yang, "Current and future clinical applications for optical imaging of cancer: from intraoperative surgical guidance to cancer screening," Seminars in Oncology, vol. 38, no. 1, pp. 109-118, 2011.

[3] T. Kim, N. Lee, D. R. Arifin et al., "In vivo micro-CT imaging of human mesenchymal stem cells labeled with gold-poly-Llysine nanocomplexes," Advanced Functional Materials, vol. 27, no. 3, p. 1604213, 2017.

[4] K. Peldschus and H. Ittrich, "Magnetic resonance imaging of metastases in xenograft mouse models of cancer," Methods in Molecular Biology, vol. 1070, p. 213, 2014.

[5] K. Kajary and Z. Molnár, "The role of 18F-FDG PET/CT before and after the treatment of multiple myeloma: our clinical experience," Advances in Modern Oncology Research, vol. 3, no. 1, p. 20, 2017.

[6] C. Vangestel, W. C. Van, G. Mees et al., "Single-photon emission computed tomographic imaging of the early time course of therapy-induced cell death using technetium $99 \mathrm{~m}$ tricarbonyl His-annexin A5 in a colorectal cancer xenograft model," Molecular Imaging, vol. 11, no. 2, p. 135, 2012.

[7] J. K. Willmann, A. M. Lutz, R. Paulmurugan et al., "Dualtargeted contrast agent for US assessment of tumor angiogenesis in vivo," International Journal of Medical Radiology, vol. 248, no. 3, p. 936, 2008.

[8] R. Weissleder, "Molecular imaging in cancer," Science, vol. 312, no. 5777, pp. 1168-1171, 2006.

[9] C. P. Jin and S. Dong, "Multimodal molecular imaging in vivo," Open Nuclear Medicine Journal, vol. 2, no. 1, pp. 145-152, 2010.

[10] T. F. Massoud and S. S. Gambhir, "Molecular imaging in living subjects: seeing fundamental biological processes in a new light," Genes and Development, vol. 17, no. 5, pp. 545-580, 2003. 
[11] A. D. Vries, E. Custers, J. Lub, S. V. D. Bosch, K. Nicolay, and H. Grüll, "Block-copolymer-stabilized iodinated emulsions for use as CT contrast agents," Biomaterials, vol. 31, no. 25, pp. 6537-6544, 2010.

[12] D. B. Elrod, R. Partha, D. Danila, S. W. Casscells, and J. L. Conyers, "An iodinated liposomal computed tomographic contrast agent prepared from a diiodophosphatidylcholine lipid," Nanomedicine Nanotechnology Biology and Medicine, vol. 5, no. 1, pp. 42-45, 2009.

[13] H. Aviv and S. F. Bartling, "Radiopaque iodinated copolymeric nanoparticles for X-ray imaging applications," Biomaterials, vol. 30, no. 29, pp. 5610-5616, 2009.

[14] W. He, K. Ai, and L. Lu, "Nanoparticulate X-ray CT contrast agents," Science China Chemistry, vol. 58, no. 5, pp. 1-8, 2015.

[15] M. F. Attia, N. Anton, M. Chiper et al., "Biodistribution of $\mathrm{X}$-ray iodinated contrast agent in nano-emulsions is controlled by the chemical nature of the oily core," ACS Nano, vol. 8, no. 10, pp. 10537-10550, 2014.

[16] M. F. Attia, N. Anton, R. Akasov, M. Chiper, E. Markvicheva, and T. F. Vandamme, "Biodistribution and toxicity of X-ray iodinated contrast agent in nano-emulsions in function of their size," Pharmaceutical Research, vol. 33, no. 3, pp. 1-12, 2016.

[17] S. J. Kim, W. Xu, M. W. Ahmad et al., "Synthesis of nanoparticle CT contrast agents: in vitro and in vivo studies," Science and Technology of Advanced Materials, vol. 16, no. 5, p. 055003, 2015.

[18] O. Rabin, J. M. Perez, J. Grimm, G. Wojtkiewicz, and R. Weissleder, "An X-ray computed tomography imaging agent based on long-circulating bismuth sulphide nanoparticles," Nature Materials, vol. 5, no. 2, pp. 118-122, 2006.

[19] F. Hyafil, J. C. Cornily, J. E. Feig et al., "Noninvasive detection of macrophages using a nanoparticulate contrast agent for computed tomography," Nature Medicine, vol. 13, no. 5, pp. 636-641, 2007.

[20] J. Li, A. Chaudhary, S. J. Chmura et al., "A novel functional CT contrast agent for molecular imaging of cancer," Physics in Medicine and Biology, vol. 55, no. 15, pp. 4389-4397, 2010.

[21] D. Kim, Y. Y. Jeong, and S. Jon, "A drug-loaded aptamergold nanoparticle bioconjugate for combined CT imaging and therapy of prostate cancer," ACS Nano, vol. 4, no. 7, pp. 3689-3696, 2010.

[22] M. N. Kayyali, L. Brake, A. J. Ramsey, A. C. Wright, B. W. O'Malley, and D. D. Li, "A novel nano-approach for targeted inner ear imaging," Journal of Nanomedicine and Nanotechnology, vol. 8, no. 4, 2017.

[23] D. Choi, S. Jeon, G. Y. Dong et al., "Iodinated echogenic glycol chitosan nanoparticles for X-ray CT/US dual imaging of tumor," Nanotheranostics, vol. 2, no. 2, pp. 117-127, 2018.

[24] L. Yue, J. Wang, Z. Dai et al., "A pH-responsive, selfsacrificial nanotheranostic agent for potential in vivo and in vitro dual modal MRI/CT imaging, real-time and in-situ monitoring of cancer therapy," Bioconjugate Chemistry, vol. 28, no. 2, pp. 400-409, 2017.

[25] J. Beik, M. Jafariyan, A. Montazerabadi et al., "The benefits of folic acid-modified gold nanoparticles in CT-based molecular imaging: radiation dose reduction and image contrast enhancement," Artificial Cells, vol. 7, pp. 1-9, 2017.

[26] J. Zhu, W. Sun, J. Zhang et al., "Facile formation of goldnanoparticle-loaded $\gamma$-polyglutamic acid nanogels for tumor computed tomography imaging," Bioconjugate Chemistry, vol. 28, no. 11, pp. 2692-2697, 2017.

[27] W. Zhao, Z. Wang, L. Chen, C. Huang, Y. Huang, and N. Jia, “A biomimetic Au@BSA-DTA nanocomposites-based contrast agent for computed tomography imaging," Materials Science and Engineering C, vol. 78, pp. 565-570, 2017.

[28] A. Astolfo, E. Schültke, R. H. Menk et al., "In vivo visualization of gold-loaded cells in mice using X-ray computed tomography," Nanomedicine Nanotechnology Biology and Medicine, vol. 9, no. 2, p. 284, 2013.

[29] E. Schültke, R. Menk, B. Pinzer et al., "Single-cell resolution in high-resolution synchrotron X-ray CT imaging with gold nanoparticles," Journal of Synchrotron Radiation, vol. 21, no. 1, pp. 242-250, 2014.

[30] M. L. James and S. S. Gambhir, "A molecular imaging primer: modalities, imaging agents, and applications," Physiological Reviews, vol. 92, no. 2, pp. 897-965, 2012.

[31] M. Haris, S. K. Yadav, A. Rizwan et al., "Molecular magnetic resonance imaging in cancer," Journal of Translational Medicine, vol. 13, no. 1, p. 313, 2015.

[32] A. M. Blamire, "The technology of MRI-the next 10 years," British Journal of Radiology, vol. 81, no. 968, pp. 601-617, 2008.

[33] Biomarkers Definitions Working Group, "Biomarkers and surrogate endpoints: preferred definitions and conceptual framework," Clinical Pharmacology and Therapeutics, vol. 69, no. 3, pp. 89-95, 2001.

[34] P. Therasse, S. G. Arbuck, E. A. Eisenhauer et al., "New guidelines to evaluate the response to treatment in solid tumors. European Organization for Research and Treatment of Cancer, National Cancer Institute of the United States, National Cancer Institute of Canada," Journal of the National Cancer Institute, vol. 92, no. 3, pp. 205-216, 2000.

[35] M. Zhao, J. G. Pipe, J. Bonnett, and J. L. Evelhoch, "Early detection of treatment response by diffusion-weighted $1 \mathrm{H}$ NMR spectroscopy in a murine tumour in vivo," British Journal of Cancer, vol. 73, no. 1, pp. 61-64, 1996.

[36] E. C. Henning, C. Azuma, C. H. Sotak, and K. G. Helmer, "Multispectral tissue characterization in a RIF-1 tumor model: monitoring the ADC and T2 responses to single-dose radiotherapy. Part II," Magnetic Resonance in Medicine, vol. 57, no. 3, pp. 513-519, 2007.

[37] A. Dzik-Jurasz, C. Domenig, M. George et al., "Diffusion MRI for prediction of response of rectal cancer to chemoradiation," The Lancet, vol. 360, no. 9329, pp. 307-308, 2002.

[38] D. M. Koh, E. Scurr, D. Collins et al., "Predicting response of colorectal hepatic metastasis: value of pretreatment apparent diffusion coefficients," American Journal of Roentgenology, vol. 188, no. 4, pp. 1001-1008, 2007.

[39] Y. Cui, X. P. Zhang, Y. S. Sun, L. Tang, and L. Shen, “Apparent diffusion coefficient: potential imaging biomarker for prediction and early detection of response to chemotherapy in hepatic metastases," Radiology, vol. 248, no. 3, pp. $894-$ 900, 2008.

[40] J. C. Gore, H. C. Manning, C. C. Quarles, K. W. Waddell, and T. E. Yankeelov, "Magnetic resonance in the era of molecular imaging of cancer," Magnetic Resonance in Medicine, vol. 29, no. 5, pp. 587-600, 2011.

[41] M. M. Mahon, A. D. Williams, W. P. Soutter et al., "1H magnetic resonance spectroscopy of invasive cervical cancer: an in vivo study with ex vivo corroboration," $N M R$ in Biomedicine, vol. 17, no. 1, p. 1, 2004.

[42] H. C. Le, M. Lupu, K. Kotedia, N. Rosen, D. Solit, and J. A. Koutcher, "Proton MRS detects metabolic changes in hormone sensitive and resistant human prostate cancer model CWR22 and CWR22r," Magnetic Resonance in Medicine, vol. 62, no. 5, pp. 1112-1119, 2009. 
[43] C. Majós, M. Julià-Sapé, J. Alonso et al., "Brain tumor classification by proton MR spectroscopy: comparison of diagnostic accuracy at short and long TE," American Journal of Neuroradiology, vol. 25, no. 10, p. 1696, 2004.

[44] C. Majós, J. Alonso, C. Aguilera et al., "Proton magnetic resonance spectroscopy ((1)H MRS) of human brain tumours: assessment of differences between tumour types and its applicability in brain tumour categorization," European Radiology, vol. 13, no. 3, pp. 582-591, 2003.

[45] M. Julià-Sapé, I. Coronel, C. Majós et al., "Prospective diagnostic performance evaluation of single-voxel 1H MRS for typing and grading of brain tumours," NMR in Biomedicine, vol. 25, no. 4, pp. 661-673, 2012.

[46] C. Majós, J. Bruna, M. Julià-Sapé et al., "Proton MR spectroscopy provides relevant prognostic information in highgrade astrocytomas," American Journal of Neuroradiology, vol. 32, no. 1, p. 74, 2011.

[47] A. P. Prescot, A. E. Locatelli, P. F. Renshaw, and D. A. Yurgelun-Todd, "Neurochemical alterations in adolescent chronic marijuana smokers: a proton MRS study," Neuroimage, vol. 57, no. 1, p. 69, 2011.

[48] M. J. Albers, M. D. Krieger, I. Gonzalezgomez et al., "Protondecoupled 31P MRS in untreated pediatric brain tumors," Magnetic Resonance in Medicine, vol. 53, no. 1, pp. 22-29, 2005.

[49] E. B. Cady, In Vivo Cerebral 31 P Magnetic Resonance Spectroscopy, Springer, Berlin, Germany, 2012.

[50] A. M. Babsky, S. K. Hekmatyar, H. Zhang, J. L. Solomon, and N. Bansal, "Application of $23 \mathrm{Na}$ MRI to monitor chemotherapeutic response in RIF-1 tumors," Neoplasia, vol. 7, no. 7, pp. 658-666, 2005.

[51] J. Kurhanewicz, R. Bok, S. J. Nelson, and D. B. Vigneron, "Current and potential applications of clinical 13C MR spectroscopy," Journal of Nuclear Medicine, vol. 49, no. 3, p. 341, 2008.

[52] S. Hu, M. Lustig, A. Balakrishnan et al., "3D compressed sensing for highly accelerated hyperpolarized 13C MRSI with in vivo applications to transgenic mouse models of cancer," Magnetic Resonance in Medicine, vol. 63, no. 2, p. 312, 2010.

[53] S. Hu, A. Balakrishnan, R. A. Bok et al., "13C-pyruvate imaging reveals alterations in glycolysis that precede c-Myc-induced tumor formation and regression," Cell Metabolism, vol. 14, no. 1, pp. 131-142, 2011.

[54] H. Hu, K. K. Katyayan, B. A. Czeskis, E. J. Perkins, and P. Kulanthaivel, "Comparison between radioanalysis and (19)F nuclear magnetic resonance spectroscopy in the determination of mass balance, metabolism and distribution of pefloxacin," Drug Metabolism and Disposition the Biological Fate of Chemicals, vol. 45, no. 4, pp. 399-408, 2017.

[55] H. W. M. V. Laarhoven, C. J. A. Punt, Y. J. L. Kamm, and A. Heerschap, "Monitoring fluoropyrimidine metabolism in solid tumors with in vivo $19 \mathrm{~F}$ magnetic resonance spectroscopy," Critical Reviews in Oncology/Hematology, vol. 56, no. 3, pp. 321-343, 2005.

[56] A. D. Sherry and M. Woods, "Chemical exchange saturation transfer contrast agents for magnetic resonance imaging," Annual Review of Biomedical Engineering, vol. 10, pp. 391411, 2008.

[57] H. T. Ta, Z. Li, C. E. Hagemeyer et al., "Molecular imaging of activated platelets via antibody-targeted ultra-small iron oxide nanoparticles displaying unique dual MRI contrast," Biomaterials, vol. 134, pp. 31-42, 2017.
[58] M. F. Bellin, M. Vasile, and S. Morel-Precetti, "Currently used non-specific extracellular MR contrast media," European Radiology, vol. 13, no. 12, pp. 2688-2698, 2003.

[59] A. M. Mohs and Z. R. Lu, "Gadolinium(III)-based bloodpool contrast agents for magnetic resonance imaging: status and clinical potential," Expert Opinion on Drug Delivery, vol. 4, no. 2, pp. 149-164, 2007.

[60] D. Artemov, "Molecular magnetic resonance imaging with targeted contrast agents," Journal of Cellular Biochemistry, vol. 90, no. 3, pp. 518-524, 2003.

[61] A. S. Krishnan, A. A. Neves, M. M. de Backer et al., "Detection of cell death in tumors by using MR imaging and a gadolinium-based targeted contrast agent," Radiology, vol. 246, no. 3, pp. 854-862, 2008.

[62] I. Pirko, A. Johnson, B. Ciric et al., "In vivo magnetic resonance imaging of immune cells in the central nervous system with superparamagnetic antibodies," Faseb Journal, vol. 18, no. 1, pp. 179-182, 2004.

[63] M. Lepage, W. C. Dow, M. Melchior et al., "Noninvasive detection of matrix metalloproteinase activity in vivo using a novel magnetic resonance imaging contrast agent with a solubility switch," Molecular Imaging, vol. 6, no. 6, pp. 393-403, 2007.

[64] B. Yoo and M. D. Pagel, "An overview of responsive MRI contrast agents for molecular imaging," Front Bioscience, vol. 13, pp. 1733-1752, 2008.

[65] R. Lebel, B. Jastrzebska, H. Therriault et al., "Novel solubilityswitchable MRI agent allows the noninvasive detection of matrix metalloproteinase-2 activity in vivo in a mouse model," Magnetic Resonance in Medicine, vol. 60, no. 5, pp. 1056-1065, 2008.

[66] C. D. Malone, E. S. Olson, R. F. Mattrey, T. Jiang, R. Y. Tsien, and Q. T. Nguyen, "Tumor detection at 3 tesla with an activatable cell penetrating peptide dendrimer (ACPPD-Gd), a T1 magnetic resonance (MR) molecular imaging agent," PLoS One, vol. 10, no. 9, article e0137104, 2015.

[67] F. Hyafil, E. Vucic, J. C. Cornily et al., "Monitoring of arterial wall remodelling in atherosclerotic rabbits with a magnetic resonance imaging contrast agent binding to matrix metalloproteinases," European Heart Journal, vol. 32, no. 12, p. 1561, 2011.

[68] W. J. Rogers, C. H. Meyer, and C. M. Kramer, "Technology insight: in vivo cell tracking by use of MRI," Nature Clinical Practice Cardiovascular Medicine, vol. 3, no. 10, pp. 554-562, 2006.

[69] J. W. Bulte, I. D. Duncan, and J. A. Frank, "In vivo magnetic resonance tracking of magnetically labeled cells after transplantation," Journal of Cerebral Blood Flow and Metabolism, vol. 22, no. 8, pp. 899-907, 2002.

[70] H. S. Kim, J. Woo, J. H. Lee et al., "In vivo tracking of dendritic cell using MRI reporter gene, ferritin," PLoS One, vol. 10, no. 5, article e0125291, 2015.

[71] A. Khurana, H. Nejadnik, F. Chapelin et al., "Ferumoxytol: a new, clinically applicable label for stem-cell tracking in arthritic joints with MRI," Nanomedicine, vol. 8, no. 12, pp. 1969-1983, 2013.

[72] G. B. Ko, H. S. Yoon, K. Y. Kim et al., "Simultaneous multiparametric PET/MRI with silicon photomultiplier PET and ultra-high-field MRI for small-animal imaging," Journal of Nuclear Medicine, vol. 57, no. 8, pp. 1309-1315, 2016.

[73] B. Y. Yang, S. H. Moon, S. R. Seelam et al., "Development of a multimodal imaging probe by encapsulating iron oxide nanoparticles with functionalized amphiphiles for lymph 
node imaging," Nanomedicine, vol. 10, no. 12, pp. 1899-1910, 2015.

[74] M. F. Kircher, Z. A. De, J. V. Jokerst et al., "A brain tumor molecular imaging strategy using a new triple-modality MRI-photoacoustic-Raman nanoparticle," Nature Medicine, vol. 18, no. 5, p. 829, 2013.

[75] L. E. Williams, "Anniversary paper: nuclear medicine: fifty years and still counting," Medical Physics, vol. 35, no. 7, pp. 3020-3029, 2008.

[76] J. van den Hoff, "Principles of quantitative positron emission tomography," Amino Acids, vol. 29, no. 4, pp. 341-353, 2005.

[77] A. Reshef, A. Shirvan, A. Akselrod-Ballin, A. Wall, and I. Ziv, "Small-molecule biomarkers for clinical PET imaging of apoptosis," Journal of Nuclear Medicine, vol. 51, no. 6, pp. 837-840, 2010.

[78] E. Dyrberg, E. L. Larsen, H. W. Hendel, and H. S. Thomsen, "Diagnostic bone imaging in patients with prostate cancer: patient experience and acceptance of NaF-PET/CT, cholinePET/CT, whole-body MRI, bone SPECT/CT," Acta Radiologica, article 284185117751280, 2018.

[79] I. Jambor, A. Kuisma, R. Huovinen et al., "Prospective evaluation of planar bone scintigraphy, SPECT/CT, 18F NaF PET/CT and whole body 1.5T MRI for detection of bone metastases in high risk breast and prostate cancer patients," Acta Oncologica, vol. 55, no. 1, pp. 59-67, 2013.

[80] I. Jambor, A. Kuisma, S. Ramadan et al., "Prospective evaluation of planar bone scintigraphy, SPECT, SPECT/CT, $18 \mathrm{~F}-\mathrm{NaF}$ PET/CT and whole body 1.5T MRI, including DWI, for the detection of bone metastases in high risk breast and prostate cancer patients: SKELETA clinical trial," Acta Oncologica, vol. 55, no. 1, pp. 1-9, 2015.

[81] C. C. Huang, F. C. Chen, P. N. Hou et al., "Quantification of myocardial blood flow and coronary flow reserve with an innovative Tc-99m sestamibi dynamic SPECT/CT method: validation with coronary angiography in a pilot study," in Proceedings of Radiological Society of North America 2013 Scientific Assembly and Annual Meeting, Chicago, IL, USA, November 2013.

[82] P. J. Slomka, D. S. Berman, and G. Germano, "Absolute myocardial blood flow quantification with SPECT/CT: is it possible," Journal of Nuclear Cardiology, vol. 21, no. 6, pp. 1092-1095, 2014.

[83] S. G. Nekolla, C. Rischpler, and K. Nakajima, "Myocardial blood flow quantification with SPECT and conventional tracers: a critical appraisal," Journal of Nuclear Cardiology, vol. 21, no. 6, pp. 1089-1091, 2014.

[84] T. Khaing, R. Ccw, W. X. Chan, C. Hao, and S. S. Wong, "Quantification of myocardial blood flow and myocardial flow reserve with SPECT imaging technique," Journal of Nuclear Cardiology, vol. 1, pp. 1-6, 2018.

[85] F. J. Bonte, "Brain blood flow SPECT," Clinical Nuclear Medicine, vol. 24, no. 9, pp. 696-697, 1999.

[86] M. Kosel, H. Brockmann, C. Frick, A. Zobel, and T. E. Schlaepfer, "Chronic vagus nerve stimulation for treatment-resistant depression increases regional cerebral blood flow in the dorsolateral prefrontal cortex," Psychiatry Research Neuroimaging, vol. 191, no. 3, p. 153, 2011.

[87] D. G. Amen, D. V. Taylor, S. Meysami, and C. A. Raji, "Deficits in regional cerebral blood flow on brain SPECT predict treatment resistant depression," Journal of Alzheimer's Disease, vol. 63, no. 2, pp. 529-538, 2018.

[88] R. Richieri, L. Boyer, J. Farisse et al., "Predictive value of brain perfusion SPECT for rTMS response in pharmacoresistant depression," European Journal of Nuclear Medicine and Molecular Imaging, vol. 38, no. 9, pp. 17151722, 2011.

[89] L. Messinis, G. Nasios, M. H. Kosmidis et al., "Efficacy of a computer-assisted cognitive rehabilitation intervention in relapsing-remitting multiple sclerosis patients: a multicenter randomized controlled trial," Behavioural Neurology, vol. 2017, Article ID 5919841, 17 pages, 2017.

[90] N. Ahmed, K. Niyaz, A. Borakati, F. Marafi, R. Birk, and S. Usmani, "Hybrid SPECT/CT imaging in the management of differentiated thyroid carcinoma," Asian Pacific Journal of Cancer Prevention, vol. 19, no. 2, p. 303, 2018.

[91] A. Mhiri, I. Slim, M. Ghezaiel et al., "The contribution of hybrid SPECT/CT imaging in the management of differentiated thyroid carcinoma," Medecine Nucleaire, vol. 36, no. 10, pp. 554-560, 2012.

[92] D. Schmidt and T. Kuwert, "Hybrid molecular imaging in differentiated thyroid carcinoma," Frontiers of Hormone Research, vol. 45, p. 37, 2016.

[93] L. Galuska, S. Barna, J. Varga, I. Garai, and E. V. Nagy, “The role of $99 \mathrm{mTc}$-DTPA retrobulbar SPECT in staging and follow-up of Graves' orbitopathy," Nuclear Medicine Review, vol. 21, no. 1, pp. 54-58, 2018.

[94] L. Galuska, A. Leovey, Z. Szucs-Farkas et al., "Imaging of disease activity in Graves' orbitopathy with different methods: comparison of (99m)Tc-DTPA and (99m)Tcdepreotide single photon emission tomography, magnetic resonance imaging and clinical activity scores," Nuclear Medicine Communications, vol. 26, no. 5, pp. 407-414, 2005.

[95] R. Zhao, J. Wang, J. Deng, W. Yang, and J. Wang, "Efficacy of (99m)Tc-EDDA/HYNIC-TOC SPECT/CT scintigraphy in Graves' ophthalmopathy," American Journal of Nuclear Medicine and Molecular Imaging, vol. 2, no. 2, p. 242, 2012.

[96] J. Sun, L. Cai, K. Zhang et al., "A pilot study on EGFRtargeted molecular imaging of PET/CT with 11C-PD153035 in human gliomas," Clinical Nuclear Medicine, vol. 39, no. 1, p. e20, 2014

[97] X. Meng, J. Yu, B. W. Loo et al., “An evaluation of molecular imaging with (11)c-PD153035 PET/CT and its association in predicting outcomes in non-small cell lung cancer treated with EGFR-TKI," Journal of Clinical Oncology, vol. 29, no. 15, p. 10543, 2011.

[98] A. Ahmadian, S. Pawar, P. Govender, J. Berman, F. L. Ruberg, and E. J. Miller, "The response of FDG uptake to immunosuppressive treatment on FDG PET/CT imaging for cardiac sarcoidosis," Journal of Nuclear Cardiology, vol. 24, no. 2, pp. 1-12, 2016.

[99] J. Kulm, E. Mittra, H. H. Guo, R. Witteles, and A. Quon, "Development of a reproducible FDG PET/CT cardiac imaging protocol for the evaluation of cardiac sarcoidosis," Acta Horticulturae, vol. 1010, no. 1010, pp. 163-168, 2013.

[100] S. Blanchflower, L. Menezes, E. Wicks, and P. Elliot, "Patient preparation for FDG PET/CT imaging to assess cardiac inflammation," Journal of Nuclear Medicine, vol. 54, no. 2, p. 2606, 2013.

[101] N. D. Volkow, J. S. Fowler, S. J. Gatley et al., "PET evaluation of the dopamine system of the human brain," Journal of Nuclear Medicine, vol. 37, no. 7, p. 1242, 1996.

[102] C. Bao, J. Wei, X. Zhao et al., "Prognostic value of fluorine18-fluorodeoxyglucose positron emission tomography/ computed tomography in primary hepatic mucosaassociated lymphoid tissue lymphoma: a case report and review of the literature," Medicine, vol. 97, no. 10, p. e9877, 2018. 
[103] A. Batouli, J. Braun, K. Singh, A. Gholamrezanezhad, B. U. Casagranda, and A. Alavi, "Diagnosis of non-osseous spinal metastatic disease: the role of PET/CT and PET/MRI," Journal of Neuro-Oncology, vol. 138, no. 2, pp. 221-230, 2018.

[104] K. Kitajima, M. Nakajo, H. Kaida et al., "Present and future roles of FDG-PET/CT imaging in the management of gastrointestinal cancer: an update," Nagoya Journal of Medical Science, vol. 79, no. 4, pp. 527-543, 2017.

[105] M. D. Wit, W. Brenner, M. Hartmann et al., "[18F]-FDGPET in clinical stage I/II non-seminomatous germ cell tumours: results of the German multicentre trial," Annals of Oncology, vol. 19, no. 9, p. 1619, 2008.

[106] T. Rudroff, J. H. Kindred, P. J. Koo, R. Karki, and J. R. Hebert, "Asymmetric glucose uptake in leg muscles of patients with multiple sclerosis during walking detected by [18F]-FDG PET/CT," NeuroRehabilitation, vol. 35, no. 4, p. 813, 2014.

[107] J. Fledelius, A. Winther-Larsen, A. A. Khalil, K. Hjorthaug, J. Frøkiær, and P. Meldgaard, "Assessment of very early response evaluation with $18 \mathrm{~F}-\mathrm{FDG}-\mathrm{PET} / \mathrm{CT}$ predicts survival in erlotinib treated NSCLC patients-A comparison of methods," American Journal of Nuclear Medicine and Molecular Imaging, vol. 8, no. 1, pp. 50-61, 2018.

[108] V. Sossi and T. J. Ruth, "Micropet imaging: in vivo biochemistry in small animals," Journal of Neural Transmission, vol. 112, no. 3, p. 319, 2005.

[109] F. Bretin, G. Warnock, M. A. Bahri et al., "Dosimetry for 6[18F]Fluoro-L-DOPA in humans based on in vivo microPET scans and ex vivo tissue distribution in mice," in Proceedings of World Molecular Imaging Congress, Dublin, Ireland, September 2012.

[110] B. L. Franc, P. D. Acton, C. Mari, and B. H. Hasegawa, "Small-animal SPECT and SPECT/CT: important tools for preclinical investigation," Journal of Nuclear Medicine, vol. 49, no. 10, pp. 1651-1663, 2008.

[111] C. Nanni, K. D. Leo, R. Tonelli et al., "FDG small animal PET permits early detection of malignant cells in a xenograft murine model," European Journal of Nuclear Medicine and Molecular Imaging, vol. 34, no. 5, pp. 755-762, 2007.

[112] F. Elvas, C. Vangestel, S. Rapic et al., "Characterization of $[99 \mathrm{~m}$ Tc]duramycin as a SPECT imaging agent for early assessment of tumor apoptosis," Molecular Imaging and Biology, vol. 17, no. 6, pp. 838-847, 2015.

[113] K. Leung, ${ }^{99 m}$ Tc-(Hydrazinonicotinic Acid-Duramycin)(Tricine) (TPPTS), Molecular Imaging and Contrast Agent Database (MICAD) [Internet], National Center for Biotechnology Information (US), Bethesda, MD, USA, 2013.

[114] A. V. Clough, S. H. Audi, S. T. Haworth, and D. L. Roerig, "Differential lung uptake of $99 \mathrm{mTc}$-hexamethylpropyleneamine oxime and $99 \mathrm{mTc}$-duramycin in the chronic hyperoxia rat model," Journal of Nuclear Medicine, vol. 53, no. 12, p. 1984, 2012.

[115] M. Laruelle, R. M. Baldwin, R. T. Malison et al., "SPECT imaging of dopamine and serotonin transporters with [123I] beta-CIT: pharmacological characterization of brain uptake in nonhuman primates," Synapse, vol. 13, no. 4, pp. 295-309, 1993.

[116] I. Mckeith, J. O’Brien, Z. Walker et al., "Sensitivity and specificity of dopamine transporter imaging with 123I-FPCIT SPECT in dementia with Lewy bodies: a phase III, multicentre study," Lancet Neurology, vol. 6, no. 4, pp. 305-313, 2007.

[117] M. Lecchi, L. Ottobrini, C. Martelli, S. A. Del, and G. Lucignani, "Instrumentation and probes for molecular and cellular imaging," Quarterly Journal of Nuclear Medicine and Molecular Imaging, vol. 51, no. 2, pp. 111-126, 2007.

[118] F. Sun and X. Liu, "Evaluation of early-stage Parkinson's disease with $\sim(99 \mathrm{~m})$ Tc-TRODAT-1 SPECT imaging," Stroke and Nervous Diseases, vol. 42, no. 9, pp. 1303-1308, 2004.

[119] A. Capello, E. Krenning, B. Bernard, J. C. Reubi, W. Breeman, and J. M. De, "111In-labelled somatostatin analogues in a rat tumour model: somatostatin receptor status and effects of peptide receptor radionuclide therapy," European Journal of Nuclear Medicine and Molecular Imaging, vol. 32, no. 11, pp. 1288-1295, 2005.

[120] S. Probst, J. Lee, T. Tu, R. Berman, and K. Friedman, “Tc-99m sestamibi SPECT/CT with volumetric reconstruction for radioguided occult lesion localization and surgical excision of melanoma metastasis," Clinical Nuclear Medicine, vol. 35, no. 3, pp. 165-167, 2010.

[121] L. Ploux and R. Mastrippolito, "In vivo radiolabel quantification in small-animal models-an approach to in vivo highresolution SPECT imaging in small laboratory animals," Europace, vol. 13, no. 3, pp. 431-437, 2011.

[122] S. R. Meikle, P. Kench, M. Kassiou, and R. B. Banati, "Small animal SPECT and its place in the matrix of molecular imaging technologies," Physics in Medicine and Biology, vol. 50, no. 22, pp. 45-61, 2005.

[123] F. Wang, W. Fang, M. Zhao et al., "Imaging paclitaxel (chemotherapy)-induced tumor apoptosis with 99mTc C2A, a domain of synaptotagmin I: a preliminary study," Nuclear Medicine and Biology, vol. 35, no. 3, pp. 359-364, 2008.

[124] A. Moscaroli, G. Jones, T. Lühmann et al., "Radiolabeled 111 In-FGF-2 is suitable for in vitro/ex vivo evaluations and in vivo imaging," Molecular Pharmaceutics, vol. 14, no. 3, pp. 639-648, 2017.

[125] L. Tang, C. Peng, B. Tang et al., "Radioiodinated small molecule tyrosine kinase inhibitor for HER2 selective SPECT imaging," Journal of Nuclear Medicine, vol. 59, no. 5, 2018.

[126] B. J. Pichler, M. S. Judenhofer, and C. Pfannenberg, "Multimodal imaging approaches: PET/CT and PET/MRI," Handbook of Experimental Pharmacology, vol. 185, no. 1, pp. 109-132, 2008.

[127] C. Glaus, R. Rossin, M. J. Welch, and G. Bao, "In vivo evaluation of $64 \mathrm{Cu}$-labeled magnetic nanoparticles as a dualmodality PET/MR imaging agent," Bioconjugate Chemistry, vol. 21, no. 4, p. 715, 2010.

[128] Q. Chen, X. Meng, P. McQuade et al., "Synthesis and preclinical evaluation of folate-NOTA-Al(18)F for PET imaging of folate-receptor-positive tumors," Molecular Pharmaceutics, vol. 13, no. 5, pp. 1520-1527, 2016.

[129] Y. Xing, J. Zhao, P. S. Conti, and K. Chen, "Radiolabeled nanoparticles for multimodality tumor imaging," Theranostics, vol. 4, no. 3, pp. 290-306, 2014.

[130] R. Pither, "PET and the role of in vivo molecular imaging in personalized medicine," Expert Review of Molecular Diagnostics, vol. 3, no. 6, pp. 703-713, 2003.

[131] X. D. Hu, L. G. Xing, and J. M. Yu, "Nuclear medical molecular imaging of tumor angiogenesis: current status and future prospects," Chinese Medical Journal, vol. 126, no. 14, pp. 2741-2746, 2013.

[132] M. M. Graham, "Clinical molecular imaging with radiotracers: current status," Medical Principles and Practice, vol. 21, no. 3, pp. 197-208, 2012.

[133] H. Choi, "Deep learning in nuclear medicine and molecular imaging: current perspectives and future directions," Nuclear Medicine and Molecular Imaging, vol. 52, no. 2, pp. 109-118, 2017. 
[134] P. Choquet and S. Salmon, "Small animal PET/SPECT-CT: first experiences at ACTREC for preclinical research," Indian Journal of Nuclear Medicine, vol. 25, p. 123, 2010.

[135] P. Aguiar, M. Herranz, and A. Ruibal, "Preliminary experience with small animal SPECT imaging on clinical gamma cameras," BioMed Research International, vol. 2014, Article ID 369509, 7 pages, 2014.

[136] A. Fenster, D. B. Downey, and H. N. Cardinal, "Threedimensional ultrasound imaging," Annual Review of Biomedical Engineering, vol. 2, no. 5, p. 457, 2000.

[137] P. N. Wells, "Ultrasound imaging," Physics in Medicine and Biology, vol. 51, no. 13, p. 548, 2006.

[138] M. Piedra, A. Allroggen, and J. R. Lindner, "Molecular imaging with targeted contrast ultrasound," Cerebrovascular Diseases, vol. 27, no. 2, pp. 66-74, 2009.

[139] J. Lindner, "Ultrasound molecular imaging," Acoustical Society of America Journal, vol. 141, no. 5, p. 4010, 2017.

[140] J. U. Voigt, "Ultrasound molecular imaging," Methods, vol. 48, no. 2, pp. 92-97, 2009.

[141] D. Cosgrove, "Ultrasound contrast agents: an overview," European Journal of Radiology, vol. 60, no. 3, pp. 324-330, 2006.

[142] K. Otani and K. Yamahara, "Development of antibodycarrying microbubbles based on clinically available ultrasound contrast agent for targeted molecular imaging: a preliminary chemical study," Molecular Imaging and Biology, vol. 13, no. 2, p. 250, 2011.

[143] M. Yang, L. Wang, Y. Guo et al., "In vivo and in vitro study of nanoscale microbubbles with G250 monoclonal antibodies targeting to renal cell carcinoma," Journal of Third Military Medical University, vol. 38, no. 2, pp. 112-117, 2016.

[144] L. I. Xiao-Yu, P. Zhang, W. Luo et al., "Preparation of ultrasound contrast agent microbubbles combining antiMUC1 monoclone antibody and evaluation of targeting capability in vitro," Progress in Modern Biomedicine, vol. 17, no. 10, pp. 1815-1817, 2017.

[145] C. Liu, Y. Yang, Z. Qiu, Y. Huang, L. Sun, and F. Yan, "Molecular ultrasound assessment of colorectal tumor angiogenesis with endoglin-targeted contrast microbubbles," in Proceedings of IEEE International Ultrasonics Symposium (IUS), pp. 1-4, Taipei, Taiwan, October 2015.

[146] J. K. Willmann, R. H. Kimura, N. Deshpande, A. M. Lutz, J. R. Cochran, and S. S. Gambhir, "Targeted contrastenhanced ultrasound imaging of tumor angiogenesis with contrast microbubbles conjugated to integrin-binding knottin peptides," Journal of Nuclear Medicine, vol. 51, no. 3, pp. 433-440, 2010.

[147] X. Wang, G. Yannik, P. Jathushan et al., “Thrombus-targeted theranostic microbubbles: a new technology towards concurrent rapid ultrasound diagnosis and bleeding-free fibrinolytic treatment of thrombosis," Theranostics, vol. 6, no. 5, p. 726, 2016.

[148] J. R. Lindner, J. Song, J. Christiansen, A. L. Klibanov, F. Xu, and K. Ley, "Ultrasound assessment of inflammation and renal tissue injury with microbubbles targeted to P-selectin," Circulation, vol. 104, no. 17, pp. 2107-2112, 2001.

[149] N. Deshpande, A. M. Lutz, Y. Ren et al., "Quantification and monitoring of inflammation in murine inflammatory bowel disease with targeted contrast-enhanced US," Radiology, vol. 262, no. 1, p. 172, 2012.

[150] J. K. Willmann, R. Paulmurugan, K. Chen et al., "US imaging of tumor angiogenesis with microbubbles targeted to vascular endothelial growth factor receptor type 2 in mice," Radiology, vol. 246, no. 2, pp. 508-518, 2008.
[151] C. Kim, R. Qin, J. S. Xu, L. V. Wang, and R. Xu, "Multifunctional microbubbles and nanobubbles for photoacoustic and ultrasound imaging," Journal of Biomedical Optics, vol. 15, no. 1, p. 010510, 2010.

[152] M. C. Cochran, J. Eisenbrey, R. O. Ouma, M. Soulen, and M. A. Wheatley, "Doxorubicin and paclitaxel loaded microbubbles for ultrasound triggered drug delivery," International Journal of Pharmaceutics, vol. 414, no. 1-2, pp. 161-170, 2011.

[153] W. Wang, G. J. Liu, X. Y. Xie et al., "Development and evaluation of lipid microbubbles targeted to alpha(v)beta(3)integrin via biotin-avidin bridge," Journal of Microencapsulation, vol. 29, no. 2, pp. 177-184, 2012.

[154] J. Liu, T. Shang, X. U. Fenfen, Z. Wang, L. I. Pan, and S. U. Lin, "Preparation of folic acid targeted liquid fluorocarbon lipid nanoparticles and application in ultrasound molecular imaging of ovarian cancer," Chinese Journal of Medical Imaging Technology, vol. 32, no. 12, pp. 1843-1847, 2016.

[155] Z. Q. Hu, B. Yang, T. Li, and J. Li, “Thyroid cancer detection by ultrasound molecular imaging with SHP2-targeted perfluorocarbon nanoparticles," Contrast Media and Molecular Imaging, vol. 2018, Article ID 8710862, 7 pages, 2018.

[156] R. C. Rintoul, A. Rawya, D. Brendan, and N. R. Carroll, "Linear endobronchial ultrasonography: a novelty turned necessity for mediastinal nodal assessment," Thorax, vol. 70, no. 2, pp. 175-180, 2015.

[157] A. Joiner, N. Massoll, T. Bartter, N. Meena, and S. Jeffus, "Endobronchial ultrasound-guided transbronchial needle aspirates (EBUS-TBNA) of mediastinal lymph nodes: assessment of proposed on-site adequacy criteria," Journal of the American Society of Cytopathology, vol. 3, no. 5, pp. S4-S5, 2014.

[158] A. Thibon and V. C. Pierre, "Principles of responsive lanthanide-based luminescent probes for cellular imaging," Analytical and Bioanalytical Chemistry, vol. 394, no. 1, pp. 107-120, 2009.

[159] Y. Yano and K. Matsuzaki, "Tag-probe labeling methods for live-cell imaging of membrane proteins," Biochimica et Biophysica Acta, vol. 1788, no. 10, pp. 2124-2131, 2009.

[160] D. Hyde, R. de Kleine, S. A. MacLaurin et al., "Hybrid FMTCT imaging of amyloid-beta plaques in a murine Alzheimer's disease model," Neuroimage, vol. 44, no. 4, pp. 1304-1311, 2009.

[161] Y. Lin, M. T. Ghijsen, H. Gao, N. Liu, O. Nalcioglu, and G. Gulsen, "A photo-multiplier tube-based hybrid MRI and frequency domain fluorescence tomography system for small animal imaging," Physics in Medicine and Biology, vol. 56, no. 15, pp. 4731-4747, 2011.

[162] H. Akimoto, H. J. Kwon, M. Ozaki, K. Yasuda, K. Honma, and Y. Ohmiya, "In vivo bioluminescence imaging of bone marrow-derived cells in brain inflammation," Biochemical and Biophysical Research Communications, vol. 380, no. 4, pp. 844-849, 2009.

[163] G. Wang, W. Cong, K. Durairaj et al., "In vivo mouse studies with bioluminescence tomography," Optics Express, vol. 14, no. 17, pp. 7801-7809, 2006.

[164] C. Ding, K. Wu, W. Wang et al., "Synthesis of a cell penetrating peptide modified superparamagnetic iron oxide and MRI detection of bladder cancer," Oncotarget, vol. 8, no. 3, pp. 4718-4729, 2017.

[165] M. A Bruckman, K. Jiang, E. J. Simpson et al., "Dual-modal magnetic resonance and fluorescence imaging of 
atherosclerotic plaques in vivo using VCAM-1 targeted tobacco mosaic virus," Nano Letters, vol. 14, no. 3, p. 1551, 2014.

[166] H. Pan, J. Myerson, X. Yang, G. Lanza, and S. A. Wickline, "Atherosclerosis endothelial activation quantification in vivo with fluorine magnetic resonance imaging and spectroscopy," Journal of Cardiovascular Magnetic Resonance, vol. 16, no. 1, p. 1, 2014.

[167] K. Chiotis, L. Saint-Aubert, I. Savitcheva et al., "Imaging invivo tau pathology in Alzheimer's disease with THK5317 PET in a multimodal paradigm," European Journal of $\mathrm{Nu}$ clear Medicine and Molecular Imaging, vol. 43, no. 9, pp. 1686-1699, 2016.

[168] S. Teipel, A. Drzezga, M. J. Grothe et al., "Multimodal imaging in Alzheimer's disease: validity and usefulness for early detection," Lancet Neurology, vol. 14, no. 10, pp. 1037-1053, 2015.

[169] D. Kim, S. Kim, S. L. Risacher et al., "A graph-based integration of multimodal brain imaging data for the detection of early mild cognitive impairment (E-MCI)," Multimodal Brain Image Analysis, vol. 8159, pp. 159-169, 2013.

[170] O. Rafael, B. H. Kopell, S. Emma, W. K. Goodman, and F. Sophia, "Multimodal neuroimaging-informed clinical applications in neuropsychiatric disorders," Front Psychiatry, vol. 7, no. 12, 2016.

[171] F. Thorsen, B. Fite, L. M. Mahakian et al., "Multimodal imaging enables early detection and characterization of changes in tumor permeability of brain metastases," Journal of Controlled Release, vol. 172, no. 3, pp. 812-822, 2013.

[172] N. Bedard, M. Pierce, A. Elnaggar, S. Anandasabapathy, A. Gillenwater, and R. Richardskortum, "Emerging roles for multimodal optical imaging in early cancer detection: a global challenge," Technology in Cancer Research and Treatment, vol. 9, no. 2, pp. 211-217, 2010.

[173] P. Arendt and H. Gerding, "Chloroquine retinopathy under inadequately weight adjusted dosage: early detection by multimodal imaging," Klinische Monatsblatter Fur Augenheilkunde, vol. 234, no. 4, pp. 543-545, 2017.

[174] C. Olsovsky, R. Cuenca, Y. S. L. Cheng, J. Wright, J. A. Jo, and K. C. Maitland, "Multimodal optical imaging for early detection of oral cancer," in Proceedings of Biomedical Optics, Fort Lauderdale, FL, USA, April 2016.

[175] Y. Tang, J. Carns, T. Quang et al., “A multimodal optical imaging platform for the early detection of gastric malignancies," Journal of Global Oncology, vol. 2, no. 3, p. 6, 2016.

[176] G. M. van Dam, G. Themelis, L. M. Crane et al., "Intraoperative tumor-specific fluorescence imaging in ovarian cancer by folate receptor- $\alpha$ targeting: first in-human results," Nature Medicine, vol. 17, no. 10, pp. 1315-1319, 2011.

[177] S. Lütje, M. Rijpkema, W. Helfrich, W. J. G. Oyen, and O. C. Boerman, "Targeted radionuclide and fluorescence dual-modality imaging of cancer: preclinical advances and clinical translation," Molecular Imaging and Biology, vol. 16, no. 6, pp. 747-755, 2014.

[178] H. Yoo, "Label-free multimodal intravascular optical imaging for cardiovascular disease," in Proceedings of Biophotonics Congress: Biomedical Optics Congress, Bangkok, Thailand, May 2018.

[179] H. U. Voss, L. A. Heier, and N. D. Schiff, "Multimodal imaging of recovery of functional networks associated with reversal of paradoxical herniation after cranioplasty," Clinical Imaging, vol. 35, no. 4, p. 253, 2011.

[180] G. Y. Wang, J. van Eijk, T. Demirakca et al., “ACC GABA levels are associated with functional activation and connectivity in the fronto-striatal network during interference inhibition in patients with borderline personality disorder," Neuroimage, vol. 147, pp. 164-174, 2017. 


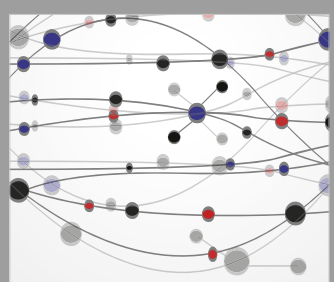

The Scientific World Journal
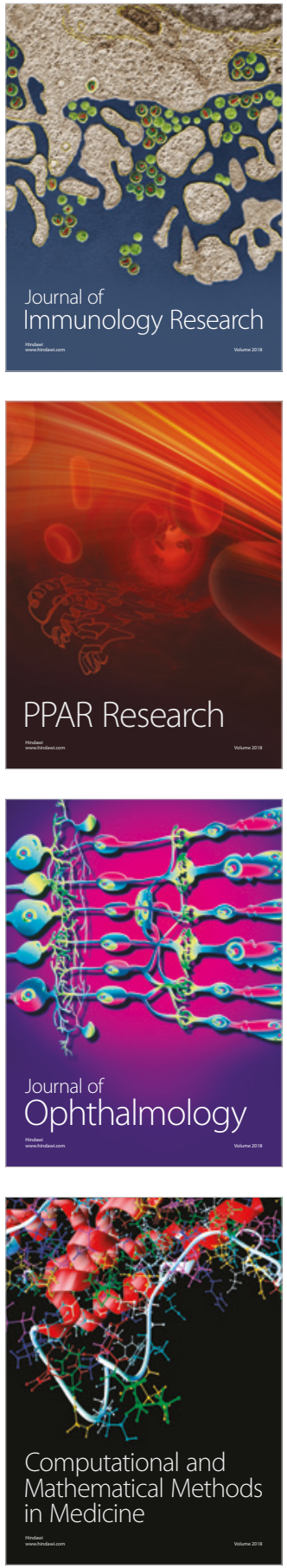

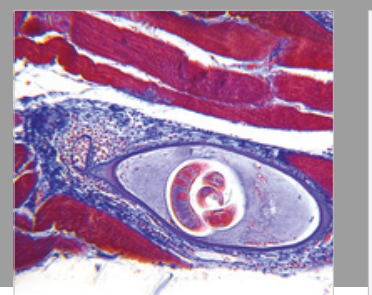

Gastroenterology Research and Practice

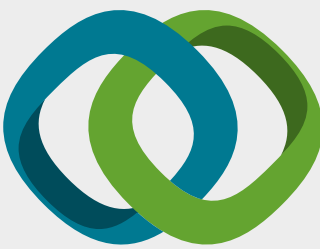

\section{Hindawi}

Submit your manuscripts at

www.hindawi.com
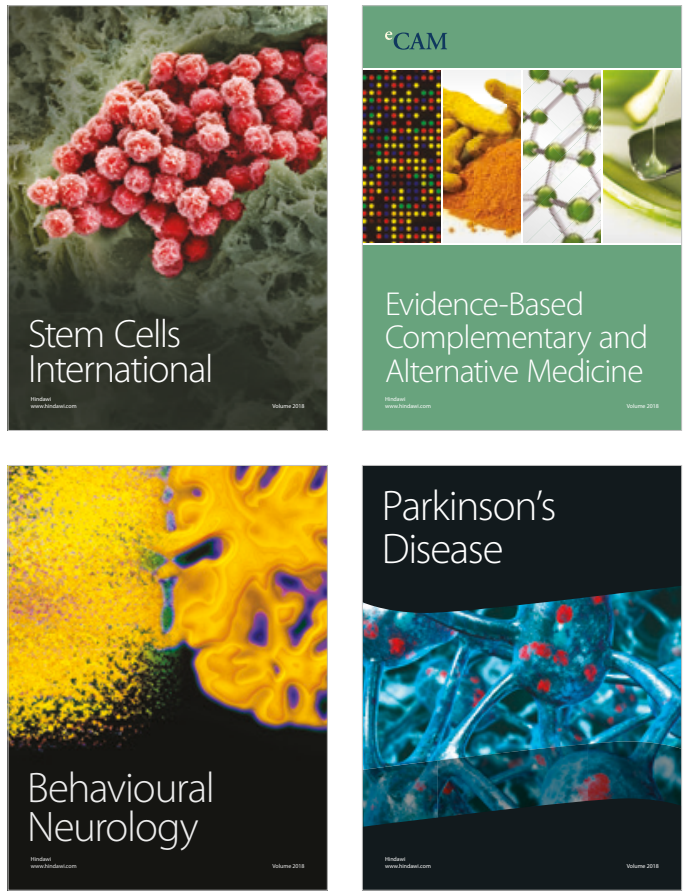

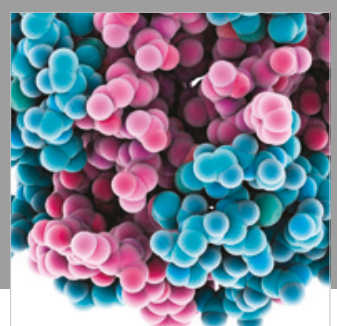

ournal of

Diabetes Research

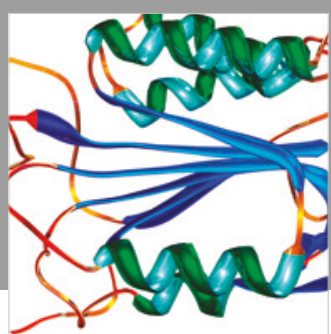

Disease Markers
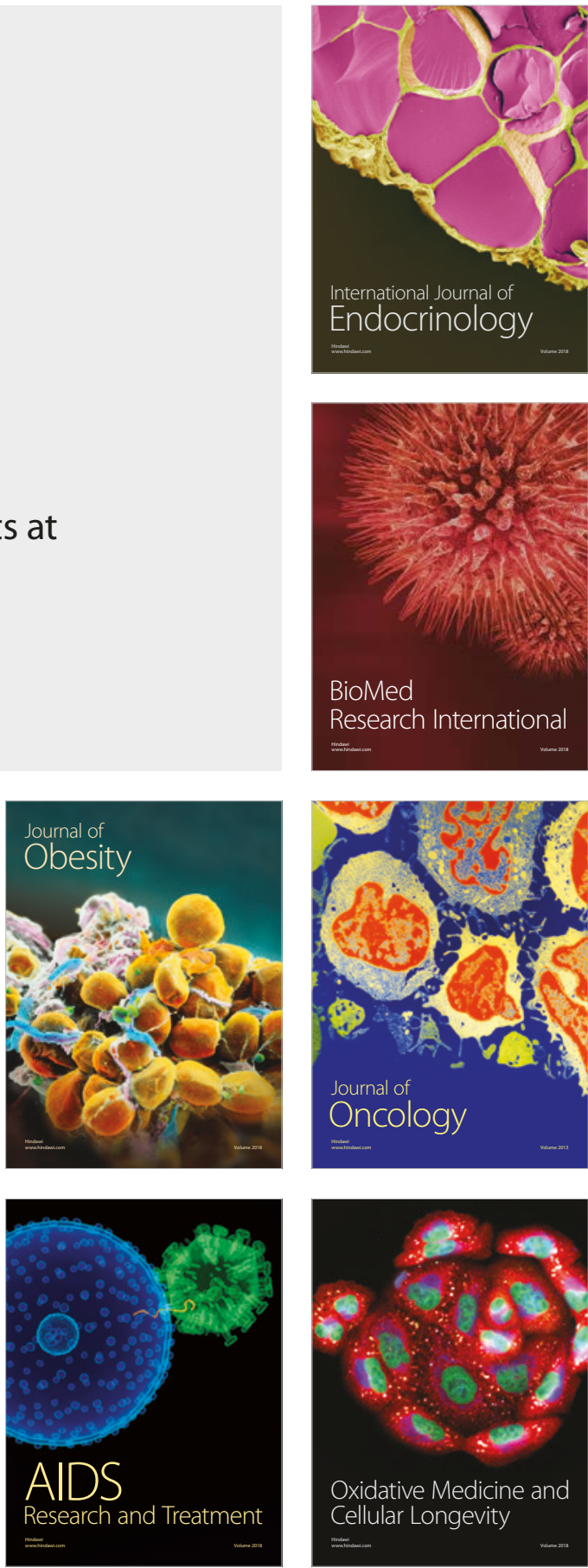